\title{
Mediastinum primary small cell neuroendocrine carcinoma
}

\author{
Hong-Lu Wang ${ }^{1,2 *}$, Zhi-Gang Sun ${ }^{1 *}$, Wei Xiao ${ }^{1}$, Liang-Ming Zhu ${ }^{1}$ \\ ${ }^{1}$ Department of Thoracic Surgery, Jinan Central Hospital Affiliated to Shandong University, Shandong University, Jinan, China \\ 2Department of Thoracic Surgery, Pingyin People's Hospital, Weifang Medical University, Shandong, China \\ *The authors have contributed equally to this paper and are co-first authors for this article.
}

Contemp Oncol (Pozn) 2016; 20 (1): 86-90 DOI: $10.5114 /$ wo.2016.58505

Neuroendocrine carcinoma, which was first reported in 1907 , is a rare disease [1]. This cancer most commonly originates in the gastrointestinal tract, although it can occur in the lungs, thymus, parathyroid gland, ovaries, and biliary system. However, mediastinal primary small cell neuroendocrine carcinoma (MPSCN) is extremely rare. Its origin is not clear [2]. The diagnosis of MPSCN requires a combination of pathological and immunohistochemical examination. The survival of patients with MPSCN was reported to be very poor, no more than 12 months [3]. Because of its rarity, only a few cases with therapy are reported in the literature. Herein, we present a case of MPSCN and the patient was alive more than three years after combined local resection, radiation, and chemotherapy.

A 36-year-old female presented to Jinan Central Hospital Affiliated to Shandong University with complaints about chest distress, dizziness, and red face for one and a half months duration. Superficial varicose veins could be seen on face, neck, double upper limbs and upper chest. These symptoms were aggravated when she was lying and relieved when she sat. $A$ chest radiograph and computed tomography (CT) scan (Fig. 1A, B, C, D, E) showed an irregular soft tissue mass located in the upper-mid mediastinum and infiltrating the right porta pulmonis, which was with uniform density and unclear border, surrounding and oppressing the superior vena cava and right innominate vein and part of the left innominate vein. All serologic and microbial tests were negative for infectious agents. Tumour markers were not elevated. A bronchoscopy was performed, which reported negative for malignancy. Patient was not found in any other parts of the lesions or lymph node enlargement. Whole-body bone imaging examination reported negative. Based on all of the above, the mass was diagnosed as a mediastinal tumour.

Because the mediastinal tumour had severely oppressed superior vena cava, right innominate vein, and part of the left innominate vein, we took resection via sternum medisect to remove the vascular compression and to make sure of diagnosis and guide further treatment. The operation showed that the tumour was located at the lower back of the left innominate vein and on the inside of right innominate vein and superior vena cava. The mass surrounded the superior vena cava and partly the left anonymous veins and right anonymous veins. There was a lymph node located behind the superior vena cava, sized $2.5 \times 2.0 \times$ $2.0 \mathrm{~cm}$. Thus the superior vena cava was disengaged partly. The tumour was partly excised surgically. Microscopic examination showed a small blue cell tumour with solid and trabecular pattern (Fig. 2). The cytoplasm was scant while the nuclear chromatin had granular appearance. Nucleoli were indistinct. Immunohistochemically the tumour cells were positive for Chromogranin A (CgA), synaptophysin, CD56, and keratin (Fig. 3A, B, C, D). The tumour was diagnosed as MPSCN pathologically.

The patient was given EP (etoposide and cisplatin) chemotherapy and local radiotherapy after operation because the small cell carcinoma is sensitive to the chemotherapy and radiotherapy and her condition improved. In the three months of follow-up, the symptoms in patients and discomfort significantly reduced compared to earlier. The chemotherapy was given six times after the operation. Meanwhile, chest CT and abdomen CT or colour Doppler ultrasound for liver, gallbladder, pancreas, spleen, and kidney were given regularly. All examinations found no signs of tumour metastasis (Fig. 4A, B, C). However, the patient complained of cough and chest distress 23 months after the operation. The chest $\mathrm{CT}$ revealed a mass in the upper right mediastinum, due to the tumour recurrence. CA125 is $79.73 \mathrm{IU} / \mathrm{ml}(0.00 \sim 35.00$ reference $\mathrm{IU} / \mathrm{ml})$. Chemotherapy used EP was given for four cycles, meanwhile local radiotherapy was given too. The patient's condition improved. However, the patient felt chest distress, asthma and breath holding, and head and facial swelling 27 months after the operation. The chest CT revealed that the mass located in the upper right mediastinum pressed on the trachea and the blood vessels locally and the right hilar lymph node was enlarged. Fibre bronchoscopy examination showed that the opening of the right bronchi was distended because of the pressure, and half of the airway was obstructed. The tumour was diagnosed as small cell carcinoma pathologically. Colour Doppler ultrasound for liver showed intrahepatic low echo. Chemotherapy using EP was given for three cycles. Tumour tissue from the previous review of chest $\mathrm{CT}$ narrowed. The patient felt better and chemotherapy and radiation therapy were given again. 

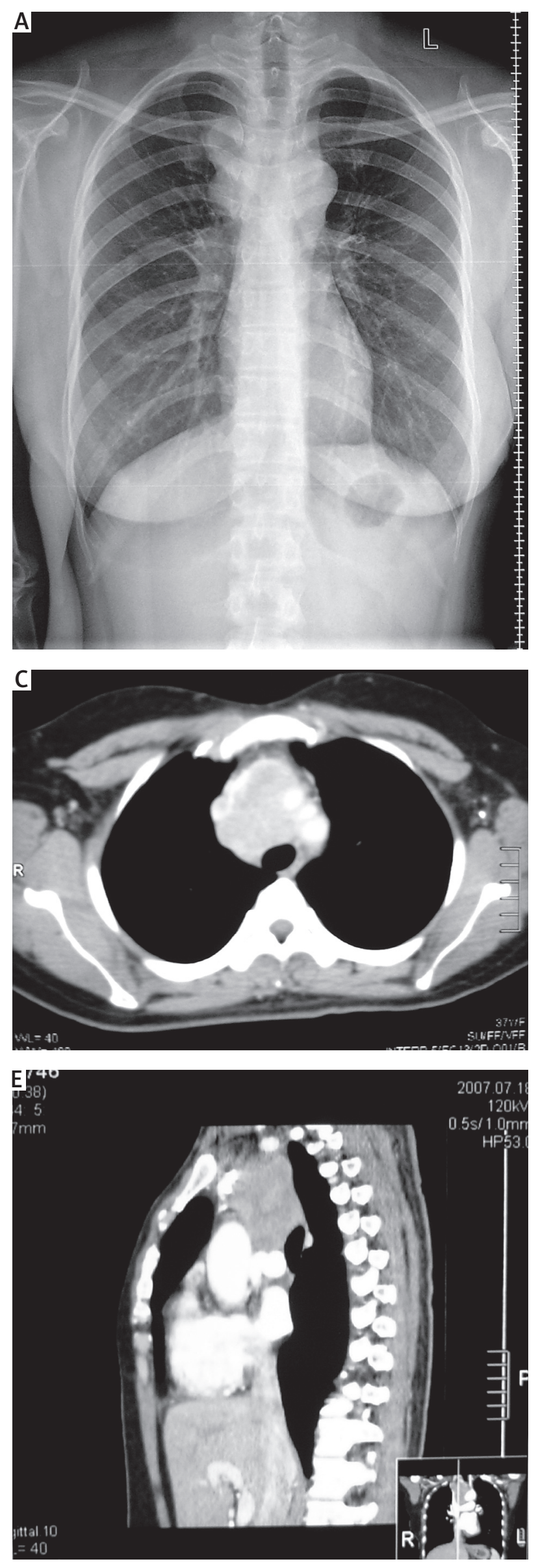
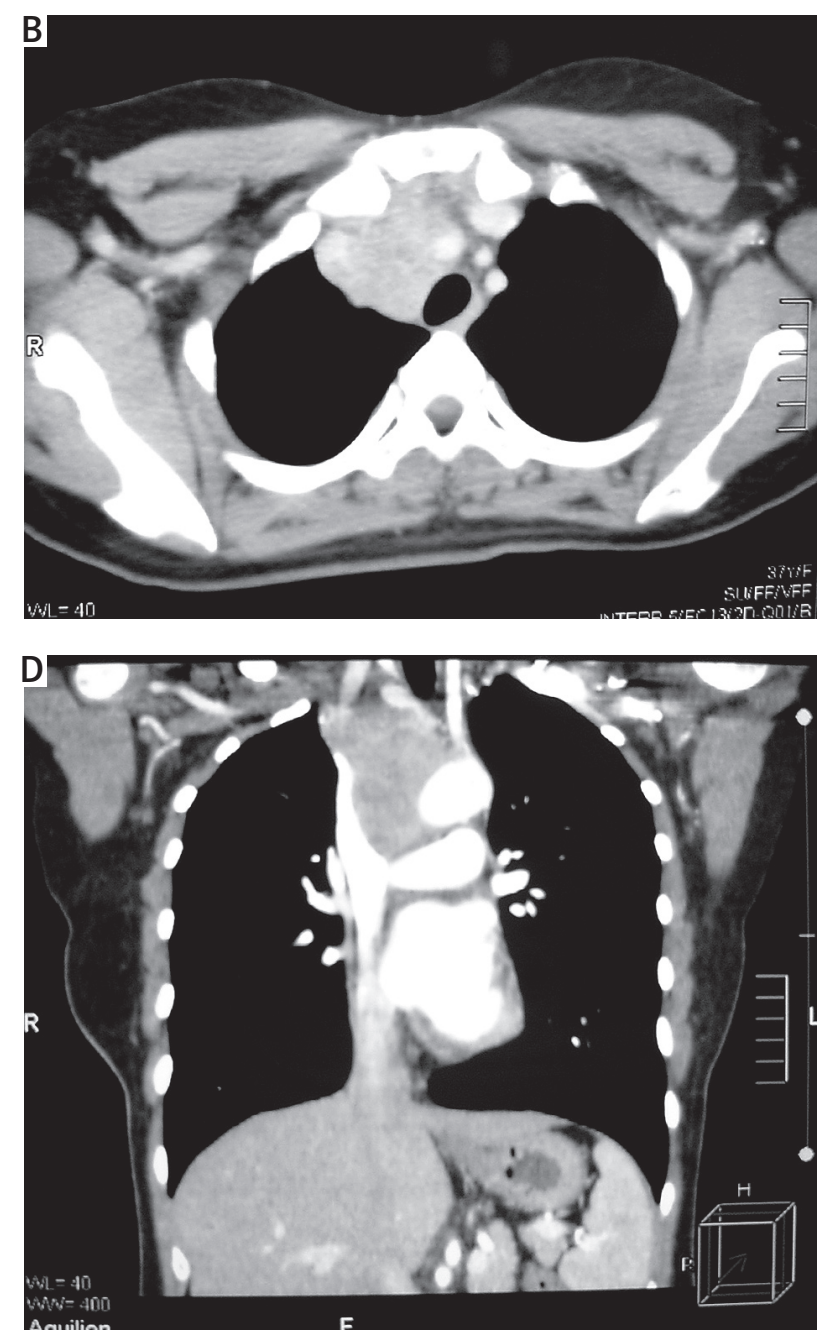

Fig. 1. Chest X-ray (A) and CT (B, C, D, E) showed an irregular soft tissue mass located in the upper-mid mediastinum and infiltrating right porta pulmonis, which was with uniform density and unclear border, surrounding and pressing on the superior vena cava and right innominate vein and part of the left innominate vein 
Unfortunately, supraclavicular lymph node enlargement was found 36 months after operation. Lymph node biopsy revealed small cell carcinoma. We gave local radiotherapy and continued the chemotherapy. CT examination showed multiple metastases in the liver and enlargement of several retroperitoneal lymph nodes 37 months after the operation. We continued to give local radiotherapy and other treatment for anti-tumour and liver protection. The patient died due to liver failure, renal insufficiency, and electrolyte disorder, which resulted from mediastinal small cell neuroendocrine carcinoma and multiple organ metastasis, such as lung, liver, and cervical lymph nodes, 39 months post operation.

Mediastinal neuroendocrine carcinomas have been reported to be more common among men, with a mean age at onset of 54 years [4]. In our study, the patient was female and 39 years old. It was rare. The tumour cells could synthesise, store, and release peptide or amine hormones such as gastrin and chromogranin. These hormones can cause specific symptoms, which are typically referred to as carcinoid syndrome and include flushing, abdominal pain, diarrhoea, asthma, and heart symptoms. Most patients have retrosternal pain, dry cough, or dyspnoea, which can occur because of mass effects. Obstruction of the superior vena cava could cause superior vena cava syndrome [2].
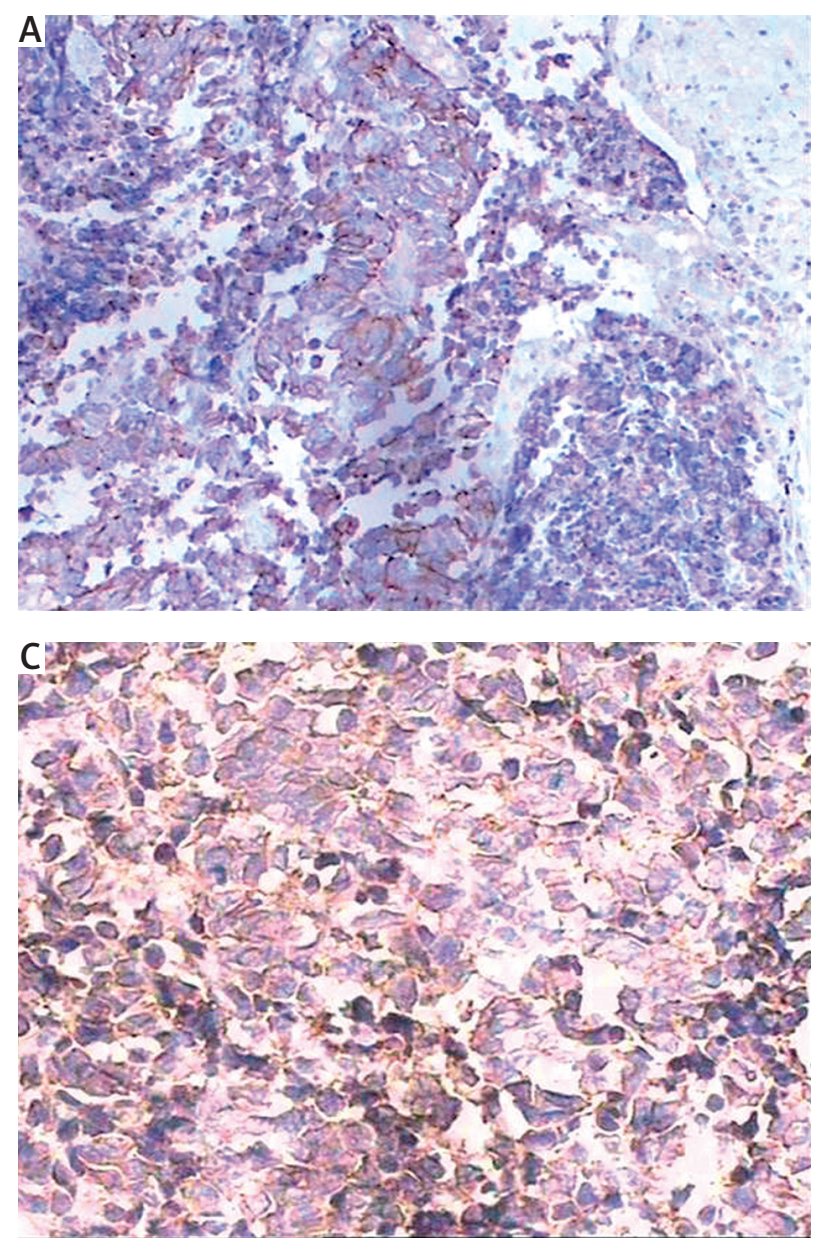

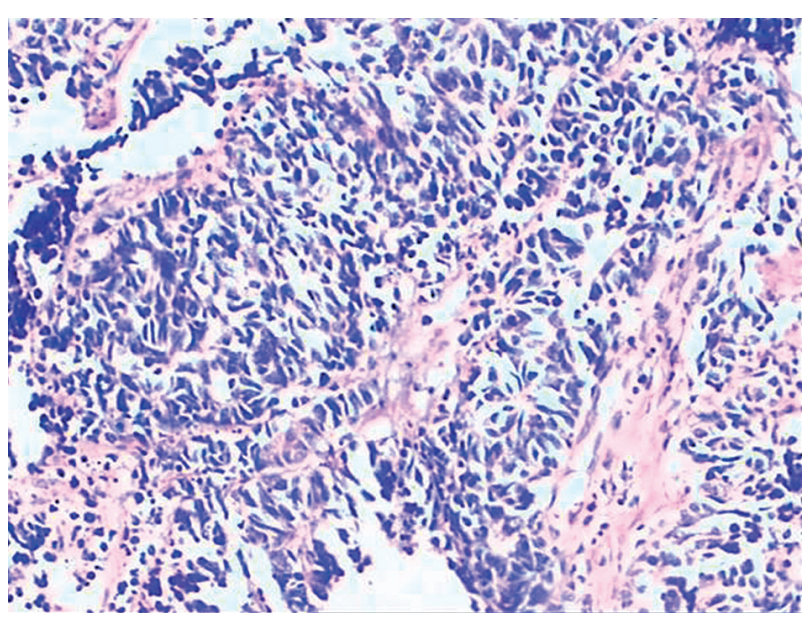

Fig. 2. Results of haematoxylin-eosin staining. Microscopic examination a small blue cell tumour in solid and trabecular pattern. The cytoplasm was scant while nuclear chromatin had granular appearance. Nucleoli were indistinct (original magnification 200x)

In our case, chest CT showed an irregular soft tissue mass located in the upper-mid mediastinum and infiltrating the right porta pulmonis, which was with uniform density and unclear border, surrounding and pressing on the superior vena cava and right innominate vein and part of the left innominate vein. Thymic tumours are mainly
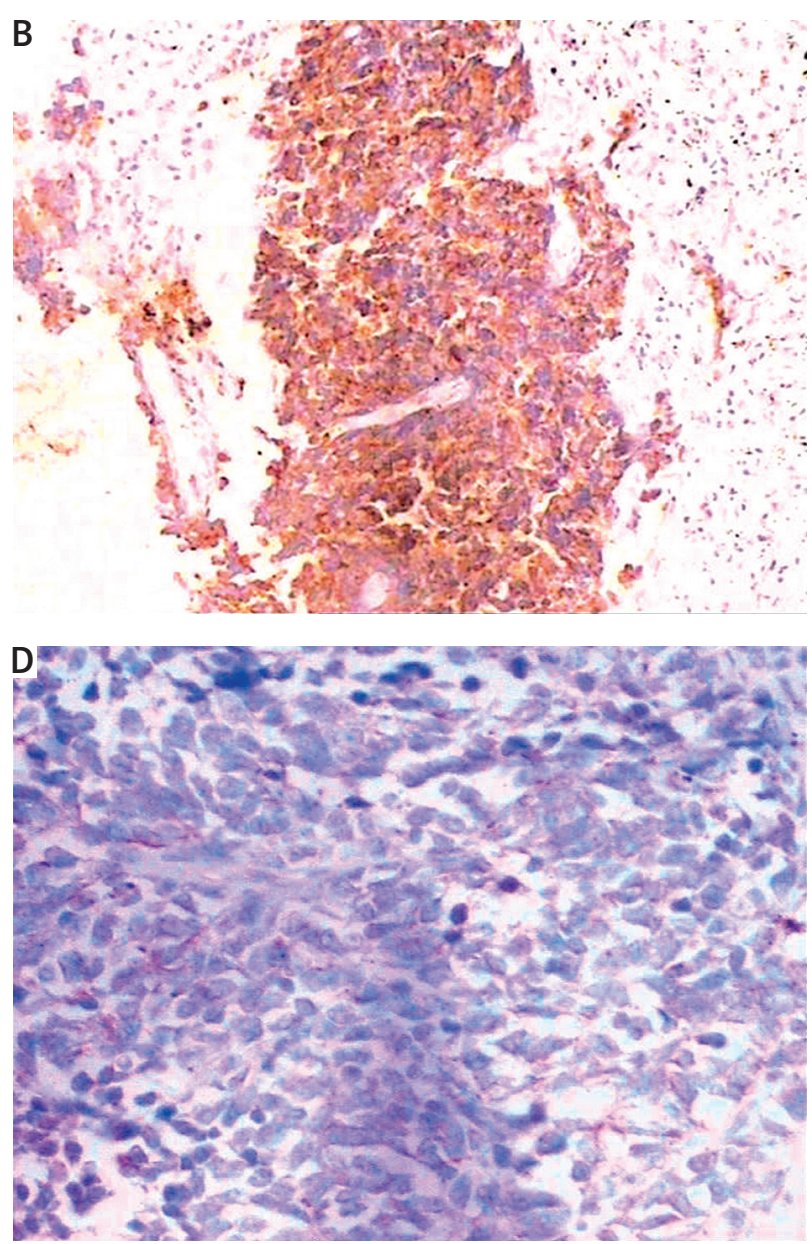

Fig. 3. Immunohistochemical analysis was positive for: A) Chromogranin A (original magnification 400x); B) synaptophysin expression (original magnification 400x); C) CD56 expression (original magnification 400x); D) keratin expression (original magnification 400x) 
located in the anterior-superior mediastinum. Thymoma shows similar imaging features to those of neuroendocrine tumours and is very difficult to identify preoperatively. Thymoma can cause myasthenia gravis and invades the lungs, cardiophrenic angle, and peritoneal cavity [5]. Mediastinal lymphoma are mainly located in the anterior or middle mediastinum. This usually involves multiple lymph nodes, which are fused together, resulting in mediastinal and paratracheal lymphadenopathy [6] Somatostatin receptor (SSTR), especially SSTR2, is overexpressed in some neuroendocrine tumours. And somatostatin receptor scintigraphy (SRS) is widely used for detection and localisation of suspected tumours, staging, follow-up, and accurate selection for somatostatin therapy. Recent studies show that radiolabelled somatostatin analogues are available for SRS. ${ }^{99 \mathrm{~m} T \mathrm{~T} \text {-depreotide displays }}$

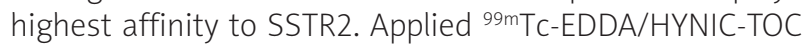
( $99 \mathrm{~m}$ Tc-TOC) has been proven to produce high-quality im-

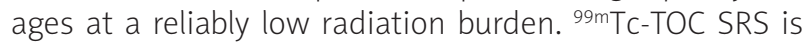
useful in the staging of neuroendocrine tumours [7]. The recent introduction of gallium-68-1,4,7,10-tetraazacyclododecane-N,N',N",N"'-tetraacetic acid (gallium-68-DOTA) compounds for positron emission tomography (PET) imaging has significantly improved the quality of imaging neuroendocrine tumours through improved resolution of PET and higher affinity of the new generation of peptides to SSTR [8]. These diagnostic methods might be effective in detecting suspected MPSCN.

The diagnosis of MPSCN requires a combination of pathological and immunohistochemical examination. Chromogranin A and synaptophysin are the main diagnostic markers of neuroendocrine carcinomas of the mediastinum. Chromogranin A is highly specific; its expression is weak or negative in nerve cell tumours and strongly positive in neuroendocrine cells and tumours. Synaptophysin is a broad-spectrum marker of neuroendocrine cells. The diagnosis of these tumours depends on the recognition of both neuroendocrine morphology (organoid pattern) and the immunohistochemical demonstration of at least one specific neuroendocrine marker $[9,10]$. In our case, the tumour was positive for chromogranin A and synaptophysin. This finding is consistent with the reports in the literature. CD56 is useful but stains a variety of other small round cell tumours. The positivity for epithelial markers, such as keratin, excluded rhabdomyosarcoma. The simultaneous expression of these markers provides a more convincing diagnosis. Based on pathological findings the tumour was considered as MPSCN. In our case we could not demonstrate any relation of the tumour with a parenchymatous organ, including the thymus. Most of the non-pulmonary neuroendocrine carcinomas arising in the mediastinum are of thymic origin. In our case, there was no evidence for involvement of the thymus, and no thymic tissue remnant was identified within the lesion.

Because of its rarity, only a few MPSCN cases with therapy are reported in the literature. In our case, the patient accepted operation and complemented with chemotherapy and radiotherapy. After operation, the initial phase condition of patient can be improved significantly. Owing to the sensitivity of the treatment, we carried out an imme-
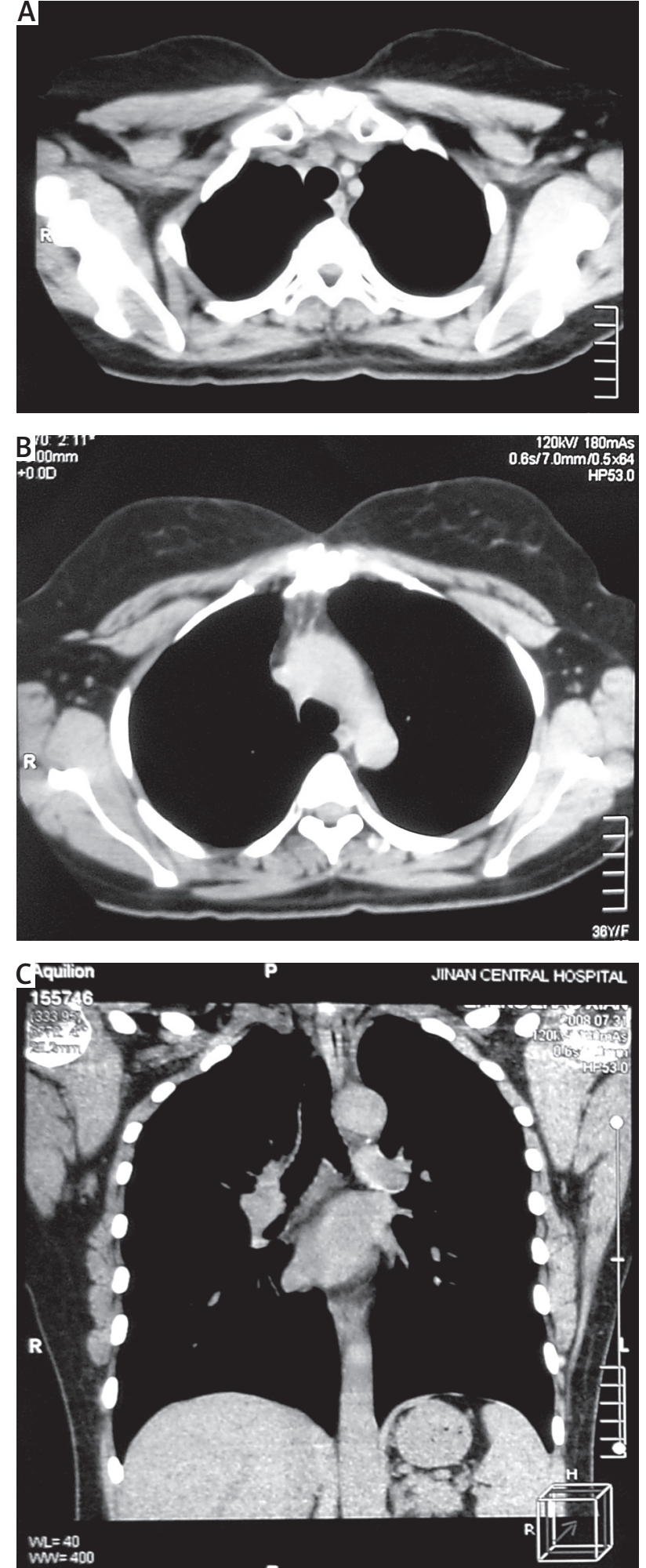

Fig. 4. Chest CT (A, B, C) showed no signs of tumour metastasis eight months after operation

diate chemotherapy and radiotherapy for several cycles, which apparently relieved the symptoms. As it is similar to small-cell-carcinoma in biological activity, tumour recurrence happened at the $23^{\text {rd }}$ month after the operation. Giving continued therapy, the treatment effect is also obvious, and symptoms were also well controlled after treatment. 
In conclusion, MPSCN is extremely rare. The diagnosis of MPSCN requires a combination of pathological and immunohistochemical examination. Operation complemented with chemotherapy and radiotherapy might alleviate the MPSCN patients' symptoms and prolong their survival. The conclusion of our study was based on one case in a single institution. We would like to study and combine with other research institutions to further confirm the validity of our conclusions.

The authors would like to thank Shan Dong University for its cooperation.

The authors declare no conflict of interest.

\section{References}

1. Dogra VS, Poblete J. Metastatic carcinoid tumor in the liver. J Clin Ultrasound 1993; 21: 639-41.

2. Li J, Xia T, Zhang W, He P, Guan Y. Primary small cell neuroendocrine carcinoma of the mediastinum: computed tomography and histopathological correlation. J Comput Assist Tomogr 2014; 38: 174-8.

3. Takanami I, Imamura T, Yamamoto Y, Yamamoto T, Kodaira S. Long survival in small-cell (neuroendocrine) carcinoma of the mediastinum. Scand J Thorac Cardiovasc Surg 1996; 30: 179-80.

4. Chaer R, Massad MG, Evans A, Snow NJ, Geha AS. Primary neuroendocrine tumors of the thymus. Ann Thorac Surg 2002; 74 : 1733-40.

5. Han J, Lee KS, Yi CA, Kim TS, Shim YM, Kim J, Kim K, Kwon OJ. Thymic epithelial tumors classified according to a newly established WHO scheme: CT and MR findings. Korean J Radiol 2003; 4: 46-53.

6. Apter S, Zaks N, Hardan I, Amitai M, Langevitz P, Livneh A. Calcification in untreated non-Hodgkin's mediastinal lymphoma. South Med J 1998; 91: 212-3.

7. Parisella MG, Chianelli M, D'Alessandria C, et al. Clinical indications to the use of $(99 \mathrm{~m})$ Tc-EDDA/HYNIC-TOC to detect somatostatin receptor-positive neuroendocrine tumors. Q J Nucl Med Mol Imaging 2012; 56: 90-8.

8. Al-Nahhas A, Win Z, Szyszko T, Singh A, Nanni C, Fanti S, Rubello D. Gallium-68 PET: a new frontier in receptor cancer imaging. Anticancer Res 2007; 27(6B): 4087-94.

9. Bajetta E, Ferrari L, Martinetti A, et al. Chromogranin A, neuron specific enolase, carcinoembryonic antigen, and hydroxyindole acetic acid evaluation in patients with neuroendocrine tumors. Cancer 1999; 86: 858-65.

10. Montero-Hadjadje M, Vaingankar S, Elias S, Tostivint H, Mahata SK, Anouar Y. Chromogranins A and B and secretogranin II: evolutionary and functional aspects. Acta Physiol (Oxf) 2008; 192: 309-24.

\section{Address for correspondence}

\section{Liang-Ming Zhu}

Department of Thoracic Surgery

Jinan Central Hospital Affiliated to Shandong University

Shandong University

Jinan 250013, China

tel. (0086)13665312567

e-mail: zlm1655@126.com

Submitted: 24.05.2014

Accepted: $\quad 8.10 .2014$ 